\title{
Dabigatran etexilato per la prevenzione del tromboembolismo venoso in pazienti sottoposti a sostituzione elettiva di anca o ginocchio
}

\author{
Valutazione di tecnologia singola presso il NICE
}

\author{
Dabigatran etexilate for the prevention of venous thromboembolism in patients \\ undergoing elective hip or knee surgery - a NICE single technology appraisal
}

\author{
M. Holmes • C. Carroll • D. Papaioannou \\ Pubblicato online: 31 luglio 2013 \\ (C) The Author(s) 2013. This article is published with open access at Springerlink.com
}

\begin{abstract}
The National Institute for Health and Clinical Excellence (NICE) invited Boehringer Ingelheim $\mathrm{GmbH}$, the manufacturer of dabigatran etexilate (DBG), to submit evidence on the clinical and cost effectiveness of this drug for the primary prevention of venous thromboembolism (VTE) in adult patients who have undergone total hip replacement (THR) or total knee replacement (TKR) surgery, as part of NICE's single technology appraisal process. The comparators were enoxaparin and fondaparinux, as identified in the scope issued by NICE. The School of Health and Related Research at the University of Sheffield was commissioned to act as the Evidence Review Group (ERG). This article provides a description of the company submission, the ERG review and NICE's subsequent decisions.

Clinical-effectiveness evidence for DBG versus enoxaparin was derived from two randomized, double-blind, noninferiority trials, one for THR and the other for TKR. Clinical-effectiveness evidence for DBG versus fondaparinux was taken from a mixed treatment comparison (MTC) and from one study. The results presented show that DBG at the licensed dose of $220 \mathrm{mg}$ and $150 \mathrm{mg}$ once daily was noninferior to enoxaparin (40 mg once daily) in terms of the primary efficacy outcome of total VTE and all-cause
\end{abstract}

Traduzione dell'articolo: Dabigatran Etexilate for the Prevention of Venous Thromboembolism in Patients Undergoing Elective Hip or Knee Surgery-A NICE Single Technology Appraisal. Pharmacoeconomics 2012;30(2):137-146.

M. Holmes ( $\varangle) \cdot$ C. Carroll · D. Papaioannou School of Health and Related Research, University of Sheffield, Regent Court, 30 Regent Street, Sheffield, S1 4DA, UK e-mail: m.w.holmes@shef.ac.uk mortality. In the MTC, fondaparinux was found to be more effective than DBG; the level of statistical significance was not reported.

The manufacturer's cost-effectiveness model estimated that at a dose of $220 \mathrm{mg}$ once a day, DBG dominated enoxaparin in both THR and TKR. At a dose of $150 \mathrm{mg}$ daily, DBG dominated enoxaparin in THR, while enoxaparin dominated DBG in TKR. At a dose of $220 \mathrm{mg}$ daily, DBG was less cost effective than fondaparinux in THR. The cost per QALY gained for fondaparinux versus DBG was $£ 11111$ (year 2008 values). At a dose of $150 \mathrm{mg}$ daily, DBG was less cost effective than fondaparinux in THR. The cost per QALY gained for fondaparinux versus DBG was $£ 6857$ (year 2008 values). In TKR, both DBG doses were dominated by fondaparinux.

There was some evidence that DBG was more cost effective than enoxaparin; however, these results were based on only one trial each in THR and TKR. Fondaparinux appeared to be more cost effective than DBG; however, this was based on indirect comparisons. NICE concluded that although there was uncertainty in the evidence base, DBG was very likely to be of equivalent clinical and cost effectiveness to enoxaparin or fondaparinux in the prevention of VTE. The NICE Appraisal Committee (AC) acknowledged that oral administration of DBG, without the need for monitoring, would reduce administration costs and that it may support adherence to treatment. Therefore, the AC concluded that DBG should be recommended as an option in the circumstances in which enoxaparin (or fondaparinux as an alternative) may be offered. 


\section{Key points per i decisori}

- La maggiore preoccupazione del Gruppo di revisione della documentazione (ERG) nasceva dal fatto che il confronto tra dabigatran etexilato (DBG) ed enoxaparina nella sostituzione totale d'anca (THR) e sostituzione totale di ginocchio (TKR) era basato soltanto su uno studio ciascuno

- Altri studi di DBG versus enoxaparina nella THR e TKR contribuirebbero a ridurre l'incertezza che circonda l'efficacia clinica ed economica di questi trattamenti

- La Commissione di valutazione (AC) del NICE ha concluso che probabilmente DBG è dotato di un'efficacia clinica ed economica equivalente a quella dei comparatori, nonostante l'incertezza nella documentazione di base

- L'AC ha riconosciuto che la somministrazione orale di DBG, che non richiede monitoraggio, può ridurre i costi di somministrazione e migliorare l'aderenza al trattamento

Il NICE (National Institute for Health and Clinical Excellence, Istituto Nazionale per l'Eccellenza Sanitaria e Clinica) è un ente indipendente con la responsabilità di fornire linee guida nazionali al NHS (National Health Service, Servizio Sanitario Nazionale) dell'Inghilterra e del Galles su diverse questioni di natura clinica e sanitaria, e anche sulla valutazione di nuove tecnologie. La STA (Single Technology Appraisal, valutazione di tecnologia singola) del NICE è una procedura specificamente progettata per valutare una singola tecnologia sanitaria, per una singola indicazione, in cui la maggior parte della documentazione di rilievo proviene dal produttore o sponsor [1]. Tipicamente, vi si fa ricorso per nuovi prodotti farmaceutici in tempi vicini al lancio. Nella STA, la documentazione è tratta soprattutto dalle informazioni inoltrate dal produttore/sponsor della tecnologia, che dovrebbe avere seguito le specifiche messe a punto dal NICE. Tali informazioni sono prese in esame dai membri di un ente esterno, indipendente dal NICE: l'ERG (Evidence Review Group, Gruppo di revisione della documentazione), che provvede a stilare un rapporto. L'AC (Appraisal Committee, Commissione di valutazione) del NICE considera la documentazione inoltrata dal produttore/sponsor e il rapporto dell'ERG unitamente a deposizioni rilasciate da esperti e altre parti interessate, per formulare indicazioni preliminari. Tutti costoro possono esprimere dei commenti su queste indicazioni preliminari, dopo di che la stessa AC si riunisce di nuovo per redigere le indicazioni finali (FAD, Final Appraisal Determination).

Questo articolo presenta un sommario del rapporto dell'ERG per la STA di dabigatran etexilato (DBG) per la prevenzione del tromboembolismo venoso (TEV) in pazien- ti sottoposti in elezione a intervento di sostituzione totale dell'anca (total hip replacement, THR) o sostituzione totale di ginocchio (total knee replacement, TKR) e del successivo sviluppo della linea guida del NICE per l'uso di DBG. Questo articolo fa parte di una serie di sommari di STA pubblicati con regolarità su PharmacoEconomics [2-12].

I particolari di tutta la documentazione rilevante per la valutazione (compresi l'ambito della valutazione, il rapporto dell'ERG, le informazioni inoltrate rispettivamente da produttore e consulenti, le FAD e i commenti relativi a ciascuno di questi elementi) sono reperibili sul sito del NICE [13].

\section{Contesto e condizione clinica}

TEV è un termine utilizzato per descrivere la trombosi venosa profonda (TVP) e l'embolia polmonare (EP). La TVP è la formazione di un trombo in una vena profonda, generalmente degli arti inferiori. Le TVP distali si verificano nelle vene profonde del polpaccio e sono quelle più comuni; le TVP prossimali si estendono alle vene poplitea, femorale superficiale, femorale e iliaca comune. Nelle TVP, trombi distaccati possono muoversi fino a raggiungere i polmoni; questa condizione è definita EP. L'EP può causare morte improvvisa; i sopravvissuti spesso necessitano di terapia intensiva, e il recupero può richiedere diversi mesi. Gli interventi elettivi di THR e di TKR sono entrambi associati a un aumento del rischio di TEV postoperatorio.

Nel National Joint Registry (NJR) [14] è registrato il numero di procedure di THR e TKR effettuate in Inghilterra e Galles ogni anno. Nel NJR, per il periodo 2009-10, sono registrate complessivamente 163.940 procedure THR e TKR. Il registro riporta i tassi di mortalità a 90 giorni e quelli a 5 anni per i pazienti sottoposti a THR o TKR primaria tra l'1 aprile 2003 e il 31 dicembre 2009. I tassi di mortalità globale a 90 giorni e a 5 anni dopo THR erano $0,6 \%$ e $9,9 \%$, rispettivamente (intervalli di confidenza al $95 \%$ non riportati). I tassi di mortalità globale a 90 giorni e a 5 anni dopo TKR erano $0,4 \%$ e $9,4 \%$, rispettivamente. Il NJR riporta anche i tassi di EP per le procedure THR e TKR effettuate tra aprile 2003 e settembre 2007 [15]. I tassi di EP a 3 mesi nei pazienti sottoposti a procedura di THR primaria erano 0,5\% (IC 95\% 0,2-1,0), 0,7\% (IC 95\% 0,6-0,8), 0,7\% (IC $95 \% 0,5-0,8$ ) e $0,5 \%$ (IC $95 \% 0,5-0,6$ ) per nessun metodo di tromboprofilassi, per tromboprofilassi solo chimica, solo meccanica e chimica e meccanica combinate, rispettivamente. I tassi di EP a 3 mesi nei pazienti trattati con TKR primaria erano $0,4 \%$ (IC 95\% 0,2-0,9), 0,5\% (IC 95\% 0,5-0,6), $0,4 \%$ (IC $95 \% 0,3-0,5$ ) e $0,4 \%$ (IC 95\% 0,4-0,5) per nessun metodo di tromboprofilassi, per tromboprofilassi solo chimica, solo meccanica e chimica e meccanica combinate, rispettivamente. 


\section{La tecnologia}

DBG $\left(\right.$ Pradaxa $\left.^{\circledR}\right)$ per via orale è un inibitore diretto della trombina, cioè è un anticoagulante, indicato per la prevenzione primaria del TEV in pazienti adulti sottoposti a intervento di THR o TKR. L'autorizzazione all'immissione in commercio di DBG è stata concessa dall' Agenzia Europea del Farmaco il 18 marzo 2008. La dose raccomandata di DBG è $220 \mathrm{mg}$ una volta al giorno, assunta in due capsule da $110 \mathrm{mg}$ [16]. Il trattamento deve essere iniziato con una singola capsula per via orale entro $1-4$ ore dalla fine dell'intervento, e continuato con due capsule al giorno per un totale di 28-35 giorni in caso di THR o un totale di 10 giorni nella TKR. Una dose di $150 \mathrm{mg}$ una volta al giorno è approvata per pazienti con compromissione renale di grado lieve o moderato, per quelli con più di 75 anni di età e per i soggetti in trattamento con amiodarone [16]. Il prezzo netto di una confezione da 10 capsule $(75 \mathrm{mg})$ è $£ 21$ e quello di una confezione da 60 capsule $(110 \mathrm{mg}$ ) è $£ 126$ (valori anno 2008) [16].

In questa STA sono inclusi due comparatori: enoxaparina e fondaparinux. Enoxaparina è un'eparina a basso peso molecolare da somministrare per iniezione sottocutanea. Per i pazienti sottoposti a chirurgia ortopedica, la dose è $40 \mathrm{mg}$ somministrati circa 12 ore prima dell'intervento, quindi $40 \mathrm{mg}$ al giorno per 7-10 giorni [16]. Il prezzo netto di una siringa preriempita da $40 \mathrm{mg}(0,4 \mathrm{ml}, 4000 \mathrm{UI})$ è di $£ 4,04$ [16]. Fondaparinux è un anticoagulante sintetico somministrato mediante iniezione sottocutanea di $2,5 \mathrm{mg}$ 6 ore dopo l'intervento, quindi $2,5 \mathrm{mg}$ al giorno per 5-9 giorni [16]. Il prezzo netto di una siringa preriempita da $2,5 \mathrm{mg}$ $(0,5 \mathrm{ml})$ è di $£ 6,41$ [16]. Il costo annuo stimato di ciascun farmaco è il seguente: (i) DBG, $£ 134$ (THR) e $£ 44$ (TKR); (ii) enoxaparina, $£ 38,38$; e (iii) fondaparinux, $£ 51,28$.

\section{La review del gruppo indipendente di revisione della documentazione (Evidence Review Group, ERG)}

Il produttore ha inviato al NICE la documentazione relativa all'uso di DBG (per l'indicazione approvata) nella prevenzione primaria del TEV in pazienti adulti sottoposti a intervento elettivo di THR o TKR. I comparatori erano enoxaparina e fondaparinux, identificati in accordo con le specifiche emesse dal NICE [17]. Il rapporto dell'ERG comprendeva una revisione critica dei dati di efficacia clinica ed economica della tecnologia contenuti nella documentazione inviata dal produttore (manufacturer submission, MS) al NICE. La review perseguiva tre scopi:

- valutare se la MS era conforme alle specifiche emesse dal NICE;

- valutare se l'interpretazione e l'analisi dei dati condotte dal produttore erano appropriate;
- indicare l'esistenza di altre fonti di informazione o di interpretazioni alternative che potessero aiutare il NICE a emettere la linea guida.

L'ERG ha condotto un dettagliato esame critico della MS. Nell' ambito di questo processo l'ERG ha avuto l'opportunità di chiedere chiarimenti su punti specifici della MS, il che ha comportato l'invio di ulteriori informazioni da parte del produttore.

\subsection{Documentazione dell'efficacia clinica}

La MS comprendeva una revisione sistematica della letteratura sugli effetti di DBG. Lo scopo era dimostrare che DBG era non inferiore alle terapie disponibili, principalmente enoxaparina, nella prevenzione dei TEV totali nei pazienti sottoposti a chirurgia THR e TKR. La review ha identificato tre trials di confronto diretto (head-to-head), di fase III, a più bracci, randomizzati, in doppio cieco, controllati, di non-inferiorità: RENOVATE [18] in pazienti sottoposti a chirurgia THR e RE-MODEL [19] e RE-MOBILIZE [20] in quelli sottoposti a chirurgia TKR. RE-NOVATE [18] e REMODEL [19] avevano confrontato l'efficacia e sicurezza di DBG alle dosi di $220 \mathrm{mg}$ e $150 \mathrm{mg}$ una volta al giorno rispetto a enoxaparina $40 \mathrm{mg}$ una volta al giorno. RE-MOBILIZE [20] aveva confrontato l'efficacia e sicurezza di DBG alle dosi di $220 \mathrm{mg}$ e $150 \mathrm{mg}$ una volta al giorno rispetto a enoxaparina $30 \mathrm{mg}$ due volte al giorno. Il follow-up in tutti e tre i trials era stato di 12-14 settimane. Due trials erano stati condotti in Europa (RE-NOVATE [18] e RE-MODEL [19]) e uno negli Stati Uniti (RE-MOBILIZE) [20]. Entrambi i trials RE-MODEL [19] (TKR) e RE-NOVATE [18] (THR) avevano riportato che entrambe le dosi di DBG erano noninferiori a enoxaparina in termini dell'outcome primario di efficacia (TEV totali/mortalità da tutte le cause): 36-41\% vs 38\% $(p<0,05)$ nel trial RE-MODEL [19] e 6-9\% vs $7 \%(p<0,001)$ nel RE-NOVATE [18]. Non erano state osservate differenze significative neppure per l'outcome primario di sicurezza (sanguinamento maggiore). Il trial REMOBILIZE [20] (TKR) aveva riportato che entrambe le dosi di DBG_cioè $220 \mathrm{mg}$ e $150 \mathrm{mg}$-erano inferiori a enoxaparina in termini di TEV totali/mortalità da tutte le cause $(31 \%$ e $34 \%$, rispettivamente, vs $25 \%$ per enoxaparina; $p<0,02$ e $p<0,01$, rispettivamente).

Usando un modello a effetti fissi, è sembrato che la metanalisi dell'outcome primario di efficacia trasversale ai tre trials mostrasse che DBG alla dose di $220 \mathrm{mg}$ una volta al giorno era non-inferiore a enoxaparina ( al giorno sia $30 \mathrm{mg}$ due volte al giorno) nel ridurre i livelli di TEV totali e di mortalità da tutte le cause tra i pazienti sottoposti a THR o a TKR [rischio relativo (RR) 1,06; IC 95\% 0,94-1,18]. Risultati analogamente non significativi sono stati generati aggregando i due trials TKR (RR 1,08; IC 95\% 0,96-1,22) e aggregando i due trials impiegando la 
stessa dose di comparatore, enoxaparina (40 $\mathrm{mg}$ una volta al giorno) [RR 0,95; IC 95\% 0,82-1,10]. DBG a entrambe le dosi era non-inferiore a enoxaparina in termini di outcomes secondari di efficacia, eventi maggiori di TEV o eventi TEV-correlati.

Anche una metanalisi dei trials RE-MODEL (TKR) e RE-NOVATE (THR) è sembrata indicare che la dose di DBG $150 \mathrm{mg}$ una volta al giorno era non-inferiore a enoxaparina relativamente agli outcomes primari (RR 1,12; IC 95\% 0,98-1,29); tuttavia, una metanalisi (modello a effetti fissi) di soltanto i due trials TKR (RE-MODEL e REMOBILIZE) [RR 1,19; IC 95\% 1,06-1,33; $p \leq 0,01$ ] e di tutti e tre i trials TKR e THR aggregati (RR 1,20; IC 95\% $1,08-1,34 ; p<0,001)$ aveva mostrato che la dose più bassa di DBG era inferiore a enoxaparina (sia $40 \mathrm{mg}$ una volta al giorno sia $30 \mathrm{mg}$ due volte al giorno) nel ridurre i livelli di TEV totali/mortalità da tutte le cause tra i pazienti che avevano ricevuto una chirurgia TKR o THR. Gli endpoints primari di sicurezza erano un sanguinamento maggiore e un sanguinamento clinicamente rilevante, ma non erano emerse differenze significative tra DBG e il comparatore principale, enoxaparina, in termini di qualsiasi evento avverso. Gli eventi avversi più comuni erano stati nausea, vomito, costipazione e piressia.

Infine, usando i risultati di una metanalisi condotta con la metodologia "mixed treatment comparison" (MTC) precedentemente pubblicata dal National Collaborating Centre for Acute Care (NCC-AC) [21], il produttore aveva confrontato i risultati dei trials di DBG con enoxaparina e fondaparinux per pazienti sottoposti a intervento chirurgico e a rischio di TVP. Era risultato che DBG si confrontava favorevolmente con enoxaparina, ma non con enoxaparina a durata estesa o fondaparinux, che sembravano entrambi relativamente più efficaci (il livello di significatività statistica della differenza non era riportato). Questa analisi si era resa necessaria perché fondaparinux compariva tra i principali comparatori predefiniti, ma non erano disponibili confronti diretti tra questa molecola e DBG. L'ERG aveva condotto anche un test di interazione, applicando un metodo per stimare il rapporto di due rischi relativi (RRR) [22]. Questo metodo è stato applicato anche ai risultati della metanalisi dei tre trials con la dose di DBG $220 \mathrm{mg}$ una volta al giorno $v s$ enoxaparina, e a quelli di una metanalisi dei dati relativi all'outcome primario (TEV) forniti da quattro trials che avevano confrontato fondaparinux $2,5 \mathrm{mg}$ una volta al giorno, iniziando 6 ore dopo l'intervento, con enoxaparina (RR 0,49; IC 95\% 0,42$0,59)$ [23]. Il rapporto dei due rischi relativi aveva indicato che fondaparinux era superiore a DBG nel ridurre il rischio di TEV totali (RRR 0,46; IC 95\% 0,38-0,57; $p<0,0001$ ). Ciò conferma i risultati del produttore per quel che concerne la superiorità relativa di fondaparinux nel confronto con DBG. Tuttavia, il numero globale di eventi di sanguinamento maggiore era più alto anche per fondaparinux rispetto a enoxaparina $(2,7 \%$ vs $1,7 \% ; p=0,008)[23]$.

\subsubsection{Critica e interpretazione della documentazione di efficacia clinica}

Il produttore aveva identificato tre trials ed è molto improbabile che sia stato omesso qualsiasi altro trial che avesse fornito risultati rilevanti concernenti DBG. Questi trials erano tutti di ragionevole qualità metodologica trattandosi di trials di non-inferiorità, benché fossero presenti alcuni limiti, specialmente per quel che riguarda i confronti con i trials di trattamento di riferimento, ossia i dati derivati dai trials dei trattamenti di riferimento che erano stati utilizzati per determinare le soglie di non-inferiorità. I trials avevano misurato una varietà di outcomes, appropriati e clinicamente rilevanti. Le metanalisi dei due trials considerati "pivotali" dal produttore (RE-MODEL e RE-NOVATE) dimostravano la non-inferiorità di DBG $220 \mathrm{mg}$ al giorno vs enoxaparina relativamente agli endpoints primari e secondari di efficacia e sicurezza. Tuttavia, vi era un problema riguardante la possibile combinazione dei trials riportati dal produttore. Il produttore sosteneva che i risultati del trial REMOBILIZE (TKR) dovessero essere esclusi dalla metanalisi perché questo trial aveva utilizzato un regime di dosaggio leggermente differente (la prima dose del comparatore, enoxaparina, era post-operatoria invece che pre-operatoria) e anche la durata del trattamento era diversa da quella degli altri trials. Tuttavia, l'ERG ha ritenuto che la combinazione dei due trials TKR (RE-MODEL e RE-MOBILIZE) fosse giustificata, dato che la loro popolazione presentava lo stesso rischio di TEV (molto più elevato rispetto alla THR) e che in essi la durata del trattamento era comparabile o simile (RE-MODEL, 6-10 giorni; RE-MOBILIZE, 12-15 giorni) rispetto a THR (RE-NOVATE, 28-35 giorni). Inoltre, la tempistica di somministrazione della prima dose di enoxaparina nel trial condotto in Europa, Australia e Sud Africa (RE-MODEL) non corrispondeva del tutto alla pratica clinica in UK, come sostenuto dal produttore. In un numero di casi non noto, la prima dose era stata somministrata dopo l'intervento, invece che prima, e si adeguava perciò alla pratica clinica negli USA piuttosto che a quella in UK. Pertanto, i due trials non erano così diversi come il produttore sosteneva, e quindi la loro aggregazione era legittima.

La validità esterna della documentazione clinica per UK è limitata. In tutti i trials inclusi i partecipanti erano più giovani dell'analoga popolazione UK e ciò potrebbe avere permesso all'intervento e alle terapie di confronto di manifestare una maggiore efficacia nella popolazione dei trials: le analisi di sottogruppo riportate per tutti e tre i trials hanno indicato che DBG è potenzialmente più efficace nei pazienti più giovani. Evidenze fornite da trials futuri potrebbero alterare i risultati di non-inferiorità di DBG, come suggerito dall'aggiunta nella metanalisi dei dati del trial USA REMOBILIZE. Piccole variazioni a livello degli RR potrebbero alterare le conclusioni concernenti l'efficacia clinica, 
cioè la non-inferiorità di DBG vs il principale comparatore, enoxaparina.

\subsection{La documentazione di cost-effectiveness}

La MS includeva una review della letteratura sulla costeffectiveness di DBG. Tra gli studi reperiti, nessuno affrontava il problema decisionale e il produttore, di conseguenza, aveva intrapreso una valutazione economica de novo, che aveva confrontato DBG con enoxaparina e fondaparinux. Il modello aveva stimato i costi e i QALY dalla propsettiva del NHS e dei servizi sociali alla persona in Inghilterra e Galles, secondo le linee guida del NICE per il caso di riferimento [24].

Il modello era una combinazione di due alberi decisionali e di un modello di Markov ciascuno per THR e TKR. Gli alberi decisionali rappresentavano le 10 settimane iniziali di fase acuta e il modello di Markov rappresentava la fase cronica lifetime. Un albero decisionale stimava i costi e i QALY associati agli eventi di tromboembolismo venoso e l'altro stimava i costi e i decrementi dei QALY dovuti agli eventi avversi (sanguinamenti maggiori/minori e trombocitopenia indotta da eparina). Questi due alberi decisionali operavano in modo indipendente. Lo stato di salute dei pazienti in uscita dall'albero decisionale degli eventi tromboembolici venosi era poi utilizzato come base per prevedere gli eventi a lungo termine (fase cronica) nel modello di Markov.

Dopo la procedura chirurgica iniziale, vi è una fase acuta in cui il rischio di TEV è massimo e gli eventi avversi sono massimamente probabili. Il differenziale tra gli effetti del trattamento riguarda solo questa fase. Nella fase cronica del modello, la transizione tra stati è guidata principalmente dai tassi di recidiva di TEV, benché gli stessi tassi siano applicati a tutti i bracci di trattamento. Qualsiasi costo incrementale o beneficio di salute accumulati nella fase acuta rimangono perciò relativamente costanti nell'orizzonte temporale lifetime del modello.

Per il confronto con enoxaparina, il rischio basale di eventi e il rischio relativo di DBG $v s$ enoxaparina sono stati derivati dal trial clinico appropriato (RE-NOVATE per THR e RE-MODEL per TKR).

Per il confronto con fondaparinux, il rischio basale di TVP (usata come una proxy del TEV) era stato derivato da una revisione della letteratura e da un MTC condotto dal NCC-AC [21]. Il produttore aveva condotto un'ulteriore metanalisi, utilizzando gli studi pubblicati identificati dal NCC$\mathrm{AC}$ per stimare il rischio basale di sanguinamento maggiore. I rischi relativi del trattamento $v s$ nessun trattamento sono stati derivati dallo stesso MTC e da un unico studio [25].

È ragionevole assumere che vi siano implicazioni a livello di utilizzo di risorse e di qualità della vita (quality of life, QOL) nella somministrazione di iniezioni sottocutanee (enoxaparina o fondaparinux) rispetto alla somministrazione orale (DBG). Il produttore si era correttamente reso conto che la proporzione di pazienti non in grado o non disposti ad autosomministrarsi iniezioni sottocutanee a casa propria, e che perciò avrebbero richiesto un aiuto, era un elemento chiave nell' analisi; tuttavia, aveva scelto di non includere questo elemento nel modello, così come aveva omesso il beneficio potenziale di una maggiore aderenza associata alla somministrazione orale rispetto a quella sottocutanea.

Il produttore aveva riportato i seguenti risultati. Alla dose registrata di $220 \mathrm{mg}$ una volta al giorno, DBG dominava enoxaparina sia nel setting THR che in quello TKR. Alla dose inferiore di $150 \mathrm{mg}$ al giorno, DBG dominava enoxaparina nel setting THR, mentre enoxaparina dominava DBG in quello TKR. Alla dose registrata di $220 \mathrm{mg}$ al giorno, DBG era meno costo-efficace di fondaparinux nella THR. Il costo per QALY guadagnato per fondaparinux vs DBG era $£ 11.111$ (valori anno 2008). Alla dose registrata di $150 \mathrm{mg}$ al giorno, DBG era meno costo-efficace di fondaparinux nella THR. Il costo per QALY guadagnato per fondaparinux vs DBG era $£ 6.857$ (valori anno 2008). Nel setting TKR, fondaparinux dominava entrambe le dosi di DBG.

L'ERG aveva richiesto al produttore di ripetere le analisi di costo-efficacia basandosi su una metanalisi degli studi RE-MODEL e RE-MOBILIZE (pazienti TKR). L'inclusione dello studio RE-MOBILIZE ha ribaltato i risultati, da DBG dominante a DBG dominato a entrambe le dosi.

\subsubsection{Critica e interpretazione della documentazione di cost-effectiveness}

L'analisi condotta dall'ERG del modello presentato aveva valutato l'appropriatezza e robustezza dell'approccio del produttore al modello decisionale e conseguentemente l'affidabilità dei risultati. Particolare attenzione era stata prestata alle variabili che avevano la maggiore probabilità di influire sui risultati di cost-effectiveness nel caso base.

Globalmente, l'ERG ha ritenuto che la metodologia impiegata dal produttore per costruire il modello fosse appropriata per l'analisi economica. Ciò nonostante, l'esame dell'ERG ha identificato delle lacune nella valutazione del produttore, la quale costituiva la sola fonte di dati per la cost-effectiveness di DBG per il NHS.

L'effetto del trattamento nella fase acuta si basava su trials in cui non era stata trovata una differenza tra i trattamenti statisticamente significativa; dove la differenza numerica tra i trattamenti era piccola, i costi e i benefici incrementali osservati nella fase acuta del modello erano altrettanto piccoli.

Dato che nella fase cronica non si osservano effetti del trattamento, i costi e i benefici incrementali cumulativi rimangono modesti su tutto l'orizzonte temporale lifetime del modello. I risultati incrementali osservati alla fine della fase acuta del modello erano simili come entità a quelli visti in qualsiasi momento di valutazione nella fase cronica. 
La preoccupazione dell'ERG originava dal fatto che questi modesti benefici incrementali si basavano su un solo trial ognuno per THR e TKR. Questi trials avevano indicato che DBG non era inferiore a enoxaparina. Le differenze numeriche identificate in questi trials erano piccole e ciò si riproduce nel modello in termini di piccoli costi incrementali e piccoli benefici incrementali.

Il produttore aveva argomentato che il trial REMOBILIZE non dovesse essere incluso nella metanalisi per i pazienti TKR a motivo di un regime di dosaggio e di una durata di trattamento differenti rispetto al trial RE-MODEL. Secondo l'ERG, i due trials non erano così diversi come asseriva il produttore, e di conseguenza potevano essere legittimamente aggregati. Quando l'analisi economica è stata ripetuta basandosi su una metanalisi degli studi RE-MODEL e RE-MOBILIZE, i risultati di cost-effectiveness si erano ribaltati, da DBG dominante a DBG dominato a entrambe le dosi. Pertanto, i risultati erano incerti ed esistevano scenari in cui DBG era più efficace ma anche altri in cui era meno efficace.

Il differenziale tra gli effetti del trattamento era relativo solo alla fase acuta del modello. Ciò è stato considerato ragionevole dall'ERG, dato che è in questa fase che i pazienti sono esposti al massimo rischio di TEV e di eventi avversi.

Il produttore non aveva incluso la QOL o i benefici in termini di uso di risorse o di aderenza che la somministrazione orale offre rispetto a quella sottocutanea. L'ERG ha ritenuto che questo approccio fosse conservativo.

\section{Conclusioni del rapporto dell'ERG}

La MS si basava sull'evidenza clinica per THR e TKR fornita da un trial per ciascuna. I trials sembravano essere stati condotti in modo adeguato e i risultati sembravano dimostrare che DBG era clinicamente non-inferiore a enoxaparina in una specifica popolazione di pazienti. Tuttavia, nel trial REMOBILIZE di supporto alla TKR, DBG era risultato statisticamente inferiore a enoxaparina relativamente all'outcome primario di efficacia. In un'analisi MTC fondaparinux era risultato apparentemente più efficace di DBG.

Il modello di cost-effectiveness del produttore aveva stimato che, alla dose registrata di $220 \mathrm{mg}$ al giorno, DBG dominava enoxaparina sia nella THR che nella TKR. Alla dose inferiore di $150 \mathrm{mg}$ al giorno, DBG dominava enoxaparina nella THR, mentre enoxaparina dominava DBG nella TKR. DBG era meno costo-efficace di fondaparinux nella THR a entrambi i dosaggi. Nella THR, il costo per QALY guadagnato per fondaparinux $v s$ DBG era $£ 11.111$ e $£ 6.857$ (valori anno 2008) per la dose di DBG più alta e per quella più bassa, rispettivamente. Nella TKR, entrambe le dosi di DBG erano dominate da fondaparinux.
Il produttore aveva scelto di non includere nel modello il costo di un'infermiera di supporto per effettuare le iniezioni sottocutanee o i potenziali benefici di una maggiore aderenza al trattamento con la somministrazione orale rispetto a quella sottocutanea. Era opinione dell'ERG che l'inclusione di questi costi e benefici avrebbe migliorato la costo-efficacia di DBG.

\section{Problemi metodologici chiave}

Benché l'ERG abbia identificato diversi punti discutibili nella MS, ha ritenuto che la maggior parte di essi avrebbe avuto un impatto solo modesto sulla direzione o l'ordine di grandezza dei risultati. Il punto che suscitava maggiore preoccupazione era il confronto di DBG con enoxaparina nel setting THR e TKR, in quanto basato su un unico trial per ogni setting.

Ulteriori trials di confronto tra DBG ed enoxaparina nel setting THR e TKR diminuirebbero l'incertezza che circonda l'efficacia clinica ed economica di questi trattamenti. Trials di DBG $v s$ fondaparinux rafforzerebbero le evidenze di base per questi trattamenti.

\section{Indicazioni dell'Istituto Nazionale per l'Eccellenza Sanitaria e Clinica (NICE)}

Dopo avere considerato l'evidenza di efficacia clinica resa disponibile dal produttore, dall'ERG, dagli esperti e da altre fonti di informazione, l'AC del NICE ha stilato le seguenti indicazioni nel settembre 2008: DBG, nell' ambito delle indicazioni per cui è registrato, è raccomandato come opzione per la prevenzione primaria degli eventi tromboembolici in adulti che siano stati sottoposti a chirurgia elettiva THR o TKR [13].

\subsection{Considerazioni sulla documentazione}

L'AC ha dapprima considerato la documentazione sull'efficacia clinica di DBG rispetto a quella di enoxaparina e ha discusso la trasferibilità dei trials alla pratica clinica in UK, essendo al contempo consapevole dell'esistenza di una variabilità nelle strategie di prevenzione. L'AC ha concordato che i dati dei trials RE-NOVATE e RE-MODEL, nei quali i pazienti nel braccio di controllo ricevevano enoxaparina $40 \mathrm{mg}$ una volta al giorno, potessero essere applicati alla pratica clinica in UK. L'AC ha concordato anche che lo studio RE-MOBILIZE, che aveva utilizzato un regime di dosaggio alternativo di enoxaparina $30 \mathrm{mg}$ due volte al giorno, non riflettesse il setting clinico in UK; ciò nonostante, lo studio contribuiva utilmente all'evidenza globale di base e i suoi risultati erano degni di essere considerati. 
L'AC ha anche considerato la documentazione sull'efficacia clinica di DBG rispetto a fondaparinux. L'AC era al corrente delle evidenze indirette che suggerivano che fondaparinux fosse più efficace di DBG nel ridurre gli eventi di TEV, pur essendo associato a una maggiore probabilità di sanguinamenti correlati al trattamento, rispetto a DBG. Tuttavia l'AC concordava con l'ERG che, a causa dei limiti dei dati inclusi nell' analisi, non era possibile determinare in base all' analisi MTC se fondaparinux fosse significativamente più o meno efficace di DBG. L'AC era consapevole della necessità di bilanciare la prevenzione di TEV con i possibili eventi avversi.

L'AC ha rilevato che, nel caso base del modello, DBG a entrambe le dosi era meno costoso e meno efficace di fondaparinux nella THR e più costoso e meno efficace di fondaparinux nella TKR. Tuttavia, l'AC si rendeva conto che le differenze tra gli interventi erano piccole, notando che i risultati del modello erano sensibili a variazioni nei parametri relativi all'efficacia clinica.

Inoltre, l' AC ha notato che il modello non aveva cercato di incorporare i benefici di utilità (in termini di disutilità evitata) associati alla somministrazione orale rispetto a quella sottocutanea, e che il modello aveva gestito in modo conservativo il beneficio potenziale di una maggiore aderenza a un trattamento orale in opposizione a uno sottocutaneo.

Nel complesso, tenendo presente che i dati di costo e di efficacia di DBG sono simili a quelli di enoxaparina e fondaparinux, e che alcuni benefici associati alla disponibilità di una formulazione orale non erano stati incorporati nel modello, l'AC ha concluso che DBG rappresentava un utilizzo delle risorse del NHS altrettanto costo-efficace di enoxaparina o fondaparinux.

L'AC ha concluso che, benché permanesse dell'incertezza nell'evidenza di base, con grande probabilità DBG era equivalente dal punto di vista clinico e di cost-effectiveness a enoxaparina o fondaparinux nella prevenzione del TEV. La Commissione ha riconosciuto che la somministrazione orale di DBG, che non richiede monitoraggio, potrebbe ridurre i costi di somministrazione e aumentare l'aderenza al trattamento. Pertanto, l'AC ha concluso che DBG debba essere raccomandato come un'opzione quando enoxaparina (o fondaparinux come alternativa) sono proponibili.

\section{Conclusione}

Un elemento degno di nota di questa STA è che le evidenze presentate verbalmente all' AC (cioè i benefici di DBG come trattamento orale in opposizione a sottocutaneo) sono stati presumibilmente altrettanto importanti per la FAD quanto le evidenze presentate nella documentazione inoltrata. Il produttore aveva scelto di non includere nella documentazione i potenziali benefici di utilità e quelli di un'aderenza aumentata e di costi di somministrazione ridotti associati alla via di somministrazione del farmaco, orale invece che sottocutanea. Si era trattato di una decisione ragionevole, dato che con ogni probabilità non esistevano evidenze dirette, mentre è del tutto sensato aspettarsi benefici di utilità e monetari dalla somministrazione orale opposta alla sottocutanea. L'AC del NICE ha concluso che DBG era probabilmente equivalente clinicamente e sul piano della cost-effectiveness ai comparatori, nonostante l'incertezza nelle evidenze di base, e che un farmaco orale avrebbe avuto un valore aggiunto. È difficile contestare questa logica. Tuttavia sarebbero utili, oltre a ulteriori trials per ridurre l'incertezza che circonda l'efficacia clinica ed economica di questi trattamenti, dei dati che quantifichino i benefici della somministrazione orale.

Ringraziamenti Questo progetto è stato finanziato dal National Institute for Health Research Health Technology Assessment (HTA) Programme (progetto numero 07/90/01 STA) e sarà pubblicato come parte di un compendio di articoli sull'attività degli ERG nella rivista Health Technology Assessment. Per ulteriori informazioni sul progetto si veda il sito del programma HTA (http://www.hta.ac.uk). Il sommario del presente rapporto ERG è stato redatto dopo l'esame della documentazione da parte dell' AC e non è stato sottoposto a peer-reviewers esterni da parte di PharmacoEconomics.

L'organizzazione finanziatrice è responsabile della scelta dei comparatori, della scelta degli outcomes da misurare [17] e della revisione e approvazione del manoscritto finale. L'organizzazione finanziatrice non ha avuto ruolo nella raccolta, gestione, analisi e interpretazione dei dati o nella preparazione del manoscritto.

Le vedute e le opinioni espresse sono quelle degli autori e non riflettono necessariamente quelle del NICE o del Dipartimento della Sanità.

Gli autori non hanno conflitti di interesse da dichiarare.

Open Access This article is distributed under the terms of the Creative Commons Attribution License which permits any use, distribution, and reproduction in any medium, provided the original author(s) and the source are credited.

\section{Bibliografia}

1. National Institute for Health and Clinical Excellence (NICE). Specification for manufacturer/sponsor submission of evidence: dabigatran etexilate for the prevention of venous thromboembolism after hip or knee replacement surgery in adults. London: NICE; 2006 [online]. Available from URL: http://www.nice. org.uk/nicemedia/live/11939/41391/41391.pdf [Accessed 2011 Oct 18].

2. Sculpher M. Single technology appraisal at the UK National Institute for Health and Clinical Excellence: a source of evidence and analysis for decision making internationally. Pharmacoeconomics. 2010;28(5):347-9.

3. Rodgers M, Griffin S, Paulden M, et al. Alitretinoin for severe chronic hand eczema: a NICE single technology appraisal. Pharmacoeconomics. 2010;28(5):351-62.

4. Bagust A, Greenhalgh J, Boland A, et al. Cetuximab for recurrent and/or metastatic squamous cell carcinoma of the head and neck: a NICE single technology appraisal. Pharmacoeconomics. 2010;28(6):439-48.

5. Stevenson M, Pandor A. Febuxostat for the management of hyperuricaemia in patients with gout: a NICE single technology appraisal. Pharmacoeconomics. 2011;29(2):133-40. 
6. Scotland G, Waugh N, Royle P, et al. Denosumab for the prevention of osteoporotic fractures in post-menopausal women: a NICE single technology appraisal. Pharmacoeconomics. 2011;29(11):951-61.

7. Dickson R, Bagust A, Boland A, et al. Erlotinib monotherapy for the maintenance treatment of non-small cell lung cancer after previous platinum-containing chemotherapy: a NICE single technology appraisal. Pharmacoeconomics. 2011;29(12):1051-62.

8. McKenna C, Maund E, Sarowar M, et al. Dronedarone for the treatment of atrial fibrillation: a NICE single technology appraisal. Pharmacoeconomics. 2012;30(1):35-46.

9. Yang H, Craig D, Epstein D, et al Golimumab for the treatment of psoriatic arthritis: a NICE single technology appraisal. Pharmacoeconomics. 2011;15(1 Suppl):87-96.

10. Boyers D, Jia X, Jenkinson D, et al Eltrombopag for the treatment of chronic idiopathic (immune) thrombocytopenic purpura: a NICE single technology appraisal. Pharmacoeconomics. 2012;30(6):483-95.

11. Burch J, Griffin S, McKenna C, et al Omalizumab for severe and persistent asthma in children aged 6 to 11 years: a NICE single technology appraisal. Pharmacoeconomics. 2012;30(11):9911004.

12. Whyte S, Pandor A, Stevenson M. Bevacizumab for metastatic colorectal cancer: a NICE single technology appraisal. Pharmacoeconomics. 2010;28(6):439-48.

13. National Institute for Health and Clinical Excellence. Dabigatran etexilate for the prevention of venous thromboembolism after hip or knee replacement surgery in adults. London: NICE; 2008 [online]. Available from URL: http://guidance.nice.org.uk/TA157 [Accessed 2011 Oct 18].

14. National Joint Registry. National Joint Registry for England and Wales: 7th annual report; 2010 [online]. Available from URL: http://www.njrcentre.org.uk/NjrCentre/LinkClick.aspx?fileticket= QkPI7kk6B2E\%3d\&tabid=86\&mid=523 [Accessed 2011 Oct 18].

15. National Joint Registry. National Joint Registry for England and Wales: 5th annual report; 2008 [online]. Available from URL: http://www.njrcentre.org.uk/NjrCentre/Portals/0/Documents/ England/Reports/5th\%20Annual.pdf [Accessed 2011 Oct 18].

16. Joint Formulary Committee. British national formulary. 59th ed. London: British Medical Association and Royal Pharmaceutical Society of Great Britain; 2010.
17. National Institute for Health and Clinical Excellence (NICE). Health technology appraisal: dabigatran etexilate for the prevention of venous thromboembolism after hip or knee replacement surgery in adults; final scope [online]. Available from URL: http:// www.nice.org.uk/nicemedia/live/11939/40423/40423.pdf [Accessed 2011 Oct 18].

18. Eriksson BI, Dahl OE, Rosencher N, et al, on behalf of the RENOVATE Study Group. Dabigatran etexilate versus enoxaparin for prevention of venous thromboembolism after total hip replacement: a randomised, double-blind, non-inferiority trial. Lancet. 2007;370:949-56.

19. Eriksson BI, Dahl OE, Rosencher N, et al. Oral dabigatran etexilate vs. subcutaneous enoxaparin for the prevention of venous thromboembolism after total knee replacement: the RE-MODEL randomized trial. J Thromb Haemost. 2007;5:2178-85.

20. Friedman RJ, Caprini JA, Comp PC, et al. Dabigatran etexilate versus enoxaparin in preventing venous thromboembolism following total knee arthroplasty. In: XXIst Congress of the International Society on Thrombosis and Haemostasis (ISTH). 2007 Jul 6-12; Geneva; 2007 [abstract no. O-W-051]. J Thromb Haemost. 2007; 5 Suppl 2.

21. National Collaborating Centre for Acute Care. Venous thromboembolism: reducing the risk of venous thromboembolism (deep vein thrombosis and pulmonary embolism) in patients admitted to hospital [online]. Available from URL: http://www.nice.org.uk/ nicemedia/pdf/CG92FullGuideline.pdf [Accessed 2011 Oct 18].

22. Altman DG, Bland JM. Interaction revisited: the difference between two estimates. BMJ. 2003;326:219.

23. Turpie AG, Bauer KA, Eriksson BI, et al. Fondaparinux vs enoxaparin for the prevention of venous thromboembolism in major orthopedic surgery: a meta-analysis of 4 randomized double-blind studies. Arch Intern Med. 2002;162:1833-40.

24. National Institute for Health and Clinical Excellence (NICE). Guide to the single technology appraisal (STA) process [online]. Available from URL: http://www.nice.org.uk/media/8DE/74/ STA_Process_Guide.pdf [Accessed 2011 Oct 18].

25. Eriksson BI, Lassen MR, on behalf of the PENTasaccharide in Hip-FRActure Surgery Plus (PENTHIFRA Plus) investigators. Duration of prophylaxis against venous thromboembolism with fondaparinux after hip fracture surgery. Arch Intern Med. 2003;163:1337-42. 